\title{
RECURSO PARA AUXÍLIO E MONITORAMENTO DE IDOSOS QUE MORAM SOZINHOS
}

\section{RESOURCE TO ASSISTANCE AND MONITORING OF ELDERLY PEOPLE LIVING ALONE}

Renato Antônio Chiari, Francisco Assis da Silva, Leandro Luiz de Almeida, Danillo Roberto Pereira, Mario Augusto Pazoti

Faculdade de Informática - FIPP, Universidade do Oeste Paulista - UNOESTE

e-mail: renatochiari_15@hotmail.com, \{chico, Ilalmeida, danilopereira, mario\}@unoeste.br

RESUMO - O aumento da expectativa de vida e a redução das taxas de fecundidade contribuem para um envelhecimento mundial, no Brasil o número de pessoas acima dos 60 anos passou de 3 milhões em 1960 para mais de 20 milhões em 2010, com isso outro número que vem aumentando é o de idosos que moram sozinhos, passando de 1,1 milhão em 1992 para 3,7 milhões em 2012. Este trabalho propõe a criação de um recurso tecnológico para auxílio e monitoramento de idosos que moram sozinhos. O recurso criado consiste em um aplicativo para smartphones com sistema operacional Android que deve ser instalado nos dispositivos do idoso e de seu responsável, permitindo o envio de avisos através de textos de exibição instantânea e avisos de exibição automática em datas e horários configurados pelo responsável, ele também conta com opções para realização de vídeo chamada e para consulta à localização do idoso. $O$ aplicativo também utiliza o acelerômetro do smartphone para detectar quando o idoso sofrer uma queda e avisar o responsável através de um SMS.

Palavras-chave: monitoramento de idoso; smartphone; detector de quedas; auxílio com medicamentos; acelerômetro; GPS; SMS;

ABSTRACT - The increase in life expectancy and the reduction of fertility rates contribute to a global aging, in Brazil the number of people over 60 years went from 3 million in 1960 to more than 20 million in 2010, with that another number that is increasing Is the number of elderly people living alone, from 1.1 million in 1992 to 3.7 million in 2012. This work proposes the creation of a technological resource to help and monitor the elderly who live alone. The feature created consists of an application for smartphones with Android operating system that must be installed on the devices of the elderly and its responsible, allowing the sending of warnings through texts of instantaneous display and warnings of automatic display at dates and times set by the person in charge, It also has options for making video calls and for consulting the location of the elderly. The application also uses the smartphone's accelerometer to detect when the elderly suffer

Recebido em: 22/08/2017 Revisado em: 04/09/2018 Aprovado em: 17/10/2018 a fall and notify the responsible through an SMS.

Keywords: monitoring of the elderly; smartphone; fall detector; aid with medicines; accelerometer; GPS; SMS. 


\section{INTRODUÇÃO}

A população humana está envelhecendo, o número de idosos vem aumentando em um ritmo mais acelerado em relação ao número de jovens, alguns dos motivos para este cenário atual estão na redução das taxas de fecundidade, diminuição da mortalidade e aumento da expectativa de vida. No Brasil o cenário não é diferente, segundo Bezerra, Almeida e Nóbrega-Therrien (2012, p. 156) “O número de idosos com idade acima de 60 anos passou de três milhões em 1960 para sete milhões em 1975, 14 milhões em 2002 e 20 milhões (20.590.599) em 2010, um aumento de $600 \%$ em cinquenta anos e estima-se que em 2020 tal número alcançará 32 milhões".

Dentre a população geral de idosos, outro número que vem aumentando é o de idosos que moram sozinhos. Entre 1992 e 2012, o número deles triplicou, passando de 1,1 milhão para 3,7 milhões - um aumento de $215 \%$ (COLLUCI, 2013).

Morando sozinho, o idoso mantem sua liberdade, porém são necessários cuidados e atenção por parte de familiares e amigos, pois o envelhecimento promove várias modificações no corpo que podem afetar atividades físicas e motoras, o que pode ocasionar acidentes envolvendo quedas.

As quedas com idosos são um problema que ocorre com certa frequência e que podem ocasionar importantes consequências físicas, psicológicas e sociais. Dentre as principais consequências decorrentes das quedas, encontram-se as fraturas, que parecem imputar ao idoso maior vulnerabilidade a novos episódios, independentemente de sua frequência. Entre outras consequências das quedas, encontram-se lesões na cabeça, ferimentos graves, ansiedade, depressão e o chamado "medo de cair" (medo de subsequentes quedas), que também pode acometer idosos que nunca caíram (MAIA et al., 2011).

Outro problema enfrentado pelos idosos está na ingestão de medicamentos. Geralmente os idosos necessitam tomar diversos tipos de medicamentos durante o dia, em dosagens e horários diferentes, o que pode causar confusão e um consequente uso incorreto. Além do cuidado de se tomar doses na quantidade correta é importante que os remédios sejam ingeridos dentro dos horários e intervalos orientados pelo médico. Estudos da cronofarmacologia mostram que o horário em que um remédio é ingerido pode aumentar sua eficácia no combate à doença ou provocar efeitos colaterais e até mesmo levar à morte (SANTOS, 2011).

Neste trabalho está apresentado um recurso tecnológico desenvolvido para auxílio e monitoramento de pessoas idosas que moram sozinhas, permitindo através de smartphones, que seus responsáveis possam manter contato através de avisos de texto instantâneo e vídeo chamadas, cadastrarem avisos que podem ser exibidos automaticamente em dias e horários préestabelecidos a fim de lembrar o idoso de compromissos ou que algum medicamento deve ser ingerido, disponibilizando informações para consulta de quando os avisos foram exibidos. O recurso permite também a consulta da localização atual do idoso além de informar o responsável através de um SMS quando o idoso sofrer uma queda.

As demais seções deste trabalho estão organizadas da seguinte maneira: na Seção 2 são apresentados alguns trabalhos relacionados; na Seção 3 são apresentadas a metodologia e o desenvolvimento do recurso; na Seção 4 são apresentados os experimentos realizados; na Seção 5 são apresentados os resultados obtidos nos experimentos realizados; por fim, a Seção 6 apresenta as conclusões.

\section{TRABALHOS RELACIONADOS}

Nos parágrafos a seguir, estão relacionados alguns dos trabalhos que serviram como base de conhecimento e inspiração para a criação do recurso apresentado neste trabalho.

No trabalho de Lima et al. (2013) foi desenvolvido um aplicativo para o sistema 
operacional iOS da Apple para monitoramento da prática de atividades físicas por pessoas da terceira idade. O aplicativo conta com recursos que utilizam o acelerômetro do smartphone para identificar uma interrupção na prática da atividade que pode ter sido ocasionada por um acidente envolvendo o idoso. Caso ocorra essa interrupção, o aplicativo possui a capacidade de coletar a localização atual do dispositivo através do GPS e informar um responsável sobre o ocorrido e a sua localização por meio de uma mensagem SMS. O aplicativo possui também, através de um Web Service, a opção de fornecer informações sobre a pessoa, como altura, peso e idade, além de dados sobre a atividade física, como a frequência cardíaca e o gasto calórico. Essas informações ficam a disposição para que possam ser integradas com outros softwares de terceiros.

No trabalho de Tanji et al. (2014) foi desenvolvido um aplicativo que trabalha em conjunto com o sensor Kinect da Microsoft. $O$ aplicativo reproduz ao idoso o exercício físico que deve ser executado e que foi previamente cadastrado por um profissional. O idoso acompanha o exercício, devendo reproduzir os movimentos exibidos pelo aplicativo enquanto o sensor Kinect avalia a execução feita por parte do idoso, por fim, é apresentada uma pontuação que representa o desempenho do idoso na execução do exercício.

No trabalho de Miranda e Marik (2008), os autores desenvolveram um dispositivo que fica preso junto ao corpo da pessoa que será monitorada, ele utiliza um sensor acelerômetro para detectar se houve ou não uma queda e caso a pessoa que sofreu a queda não consiga se levantar ou pedir ajuda, o sistema se encarrega de avisar um responsável por meio de uma mensagem SMS.

\section{METODOLOGIA UTILIZADA}

O recurso tecnológico desenvolvido neste trabalho consiste em um aplicativo para smartphones que utilizam o sistema operacional Android, o aplicativo deverá ficar instalado no smartphone do idoso e de seu responsável. O recurso conta com as opções de enviar mensagens de exibição instantânea ou automática em data e horário predefinidos, realizar vídeo chamadas e consultar a localização atual do idoso, além de informar ao responsável caso o idoso sofra uma queda. As figuras a seguir representam os diagramas de Casos de Uso das funcionalidades presentes no recurso. A Figura 1 apresenta o diagrama para o envio de um aviso instantâneo, onde um dispositivo envia um aviso em forma de texto que será exibido automaticamente quando o segundo dispositivo receber.

Figura 1. Diagrama de Casos e Uso do envio de um aviso instantâneo.

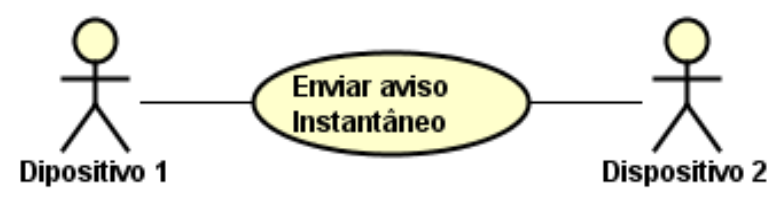

A Figura 2 apresenta o diagrama de envio de um aviso automático, em que o dispositivo do responsável envia um aviso de exibição pré-programada para o dispositivo do idoso. O aviso é exibido para o idoso automaticamente quando seu dispositivo atingir a data e a hora agendada pelo responsável, assim que o idoso confirmar a leitura do aviso, seu dispositivo envia a data e a hora de leitura para o dispositivo do responsável. 
Figura 2. Diagrama de Casos e Uso do envio de um aviso automático.

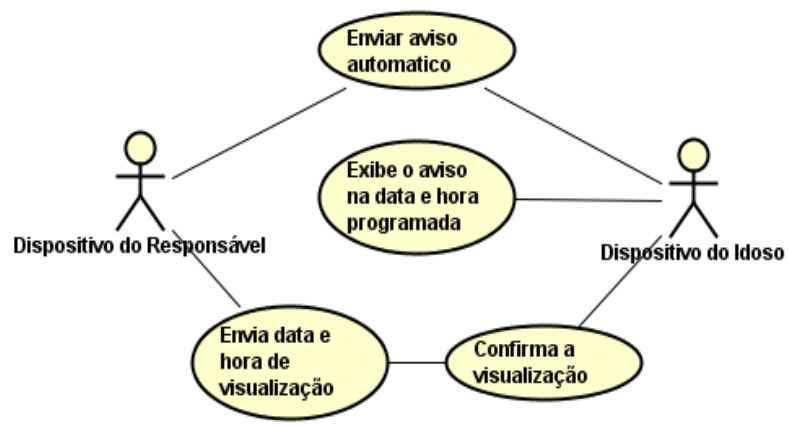

A Figura 3 apresenta o diagrama para a realização de uma vídeo chamada entre os dois dispositivos.

Figura 3. Diagrama de Casos e Uso da realização de uma vídeo chamada entre os dois dispositivos.

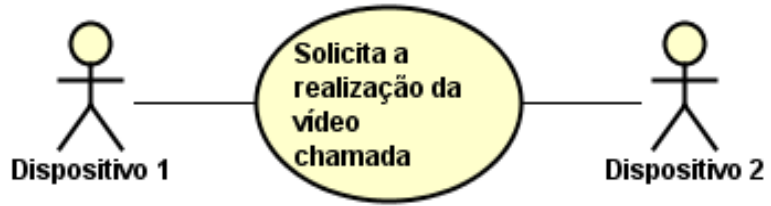

A Figura 4 apresenta o diagrama para a solicitação de consulta a localização atual do idoso através do dispositivo do responsável.

Figura 4. Diagrama de Casos e Uso da consulta à localização atual do idoso.

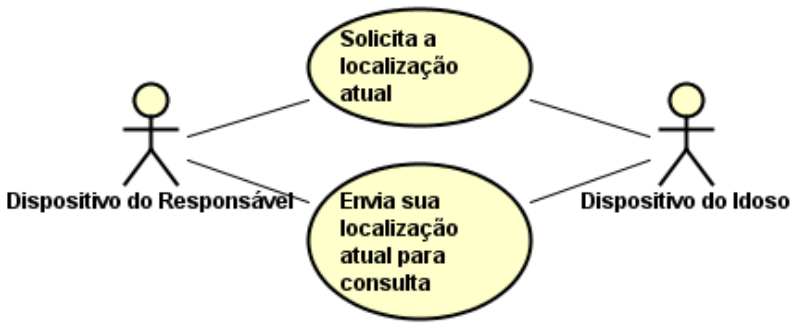

A Figura 5 apresenta o diagrama para o funcionamento do detector de quedas. 0 dispositivo do idoso monitora o acelerômetro e quando identifica uma queda avisa o responsável.
Figura 5. Diagrama de Casos e Uso do funcionamento do detector de quedas.

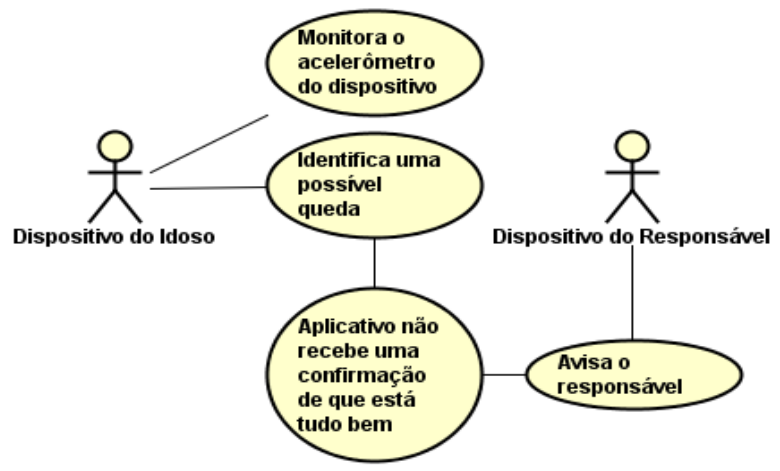

\subsection{Avisos de exibição instantânea}

O envio de avisos instantâneos pode ser executado tanto pelo responsável quanto pelo idoso, o aplicativo possui um campo onde o texto do aviso deve ser digitado, ao clicar no botão de envio, o aviso é enviado e apresentado automaticamente no outro smartphone de forma instantânea.

$O$ aviso trafega entre os dispositivos utilizando os recursos do Firebase Cloud Messaging da Google que permite a troca de informações através de um Webservice utilizando arquivos $\mathrm{JSON}^{1}$. A Figura 6 representa a forma como o aviso é exibido no dispositivo de destino, e a Figura 7 representa o arquivo JSON utilizado para o envio dos avisos instantâneos.

\footnotetext{
${ }^{1}$ JSON - É uma formatação leve de troca de dados Para seres

JSON - É uma formatação leve de troca de dados Para seres
humanos, é fácil de ler e escrever. Para máquinas, é fácil de interpretar e gerar (JSON.ORG, 2017).
} 
Figura 6. Exemplo de tela de exibição do aviso instantâneo.

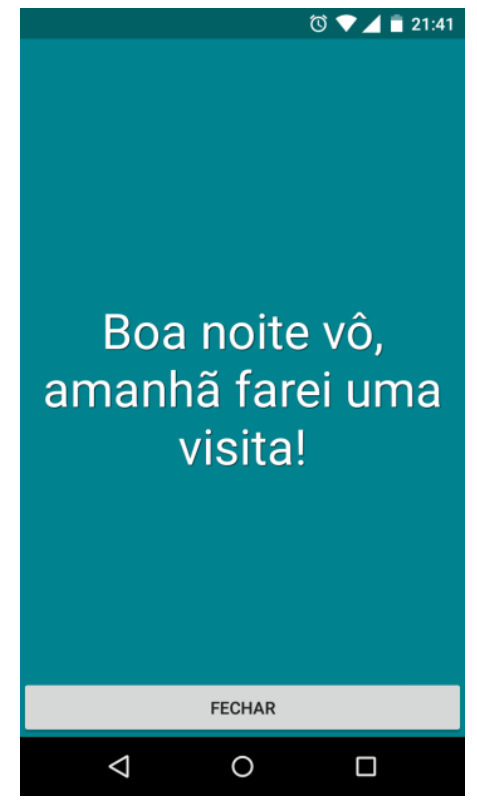

Figura 7. Estrutura do arquivo JSON para envio do aviso instantâneo.

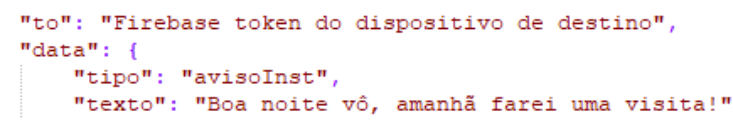

\subsection{Avisos de exibição automática}

Os avisos devem ser cadastrados utilizando o smartphone do responsável, dispensando assim a necessidade de utilizar o dispositivo do idoso. Para cadastrar o aviso, o responsável deve informar o texto que será apresentado, a data e hora que o aviso deverá ser exibido, o número de exibições e o intervalo entre cada exibição. Após finalizar o cadastro, o aviso é gravado em um banco de dados SQLite ${ }^{2}$ local, após a gravação o aviso é enviado através do Firebase Cloud Messaging para o dispositivo do idoso. Este, após receber os dados, grava em seu próprio banco de dados local, desta forma o aplicativo necessita de uma conexão com a internet apenas para trafegar as informações entre os dois smartphones, não afetando as exibições dos avisos caso não exista uma

\footnotetext{
${ }^{2}$ SQLite - É um mecanismo de banco de dados SQL incorporado. Ao contrário da maioria dos outros bancos de dados SQL, o SQLite não possui um processo de servidor separado (SQLITE.ORG, 2017).
}

conexão. Após o dispositivo do idoso receber e gravar o aviso em seu banco de dados local, o aplicativo utiliza o gerenciador de alarme do Android para programar a exibição na data e horário informado no momento do cadastro. Quando o tempo informado é alcançado, o gerenciador de alarmes dispara um evento, onde um broadcast receiver ${ }^{3}$ do aplicativo é acionado e este exibe o aviso ao idoso. A Figura 8 mostra a representação da tabela responsável pelo armazenamento do aviso, enquanto a Figura 9 apresenta a estrutura do arquivo JSON para o tráfego do aviso entre os smartphones, e a Figura 10 apresenta um exemplo da tela de exibição do aviso ao idoso.

Figura 8. Representação da tabela "aviso".

\begin{tabular}{|l|}
\hline \multicolumn{1}{|c|}{ aviso } \\
\hline - id : Integer \\
- idRef : Integer \\
- texto : Text \\
- dataProximaExibicao : Text \\
- horaProximaExibicao : Text \\
- numeroExibicoes : Integer \\
- numeroExibicoesIndetermiado : Integer \\
- intervaloEntreExibicoes : Integer \\
- tipolntervalo : Integer \\
\hline
\end{tabular}

Figura 9. Estrutura do arquivo JSON para envio do aviso automático.

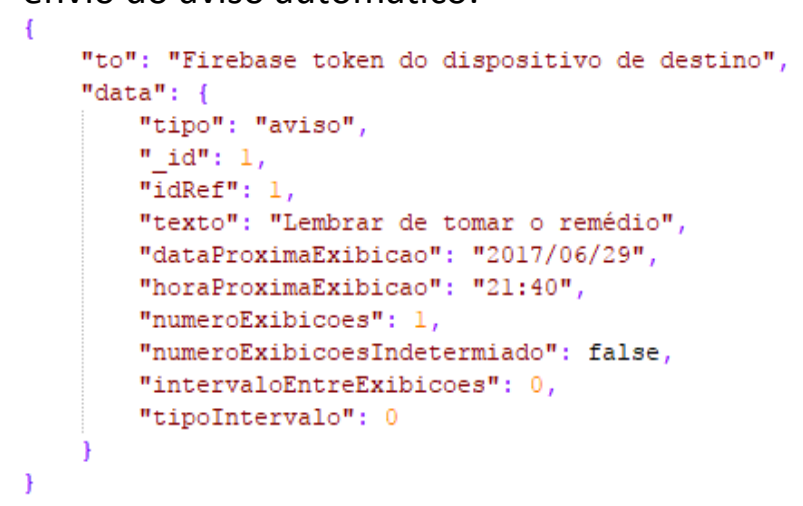

\footnotetext{
${ }^{3}$ Broadcast receiver - São componentes responsáveis por receber e tratar eventos provenientes do sistema ou de outras aplicações (SILVEIRA, 2015).
} 
Figura 10. Tela de exibição do aviso automático.

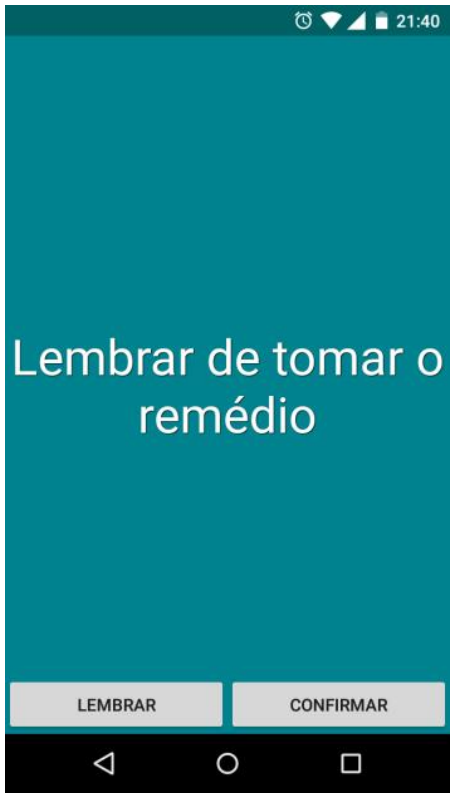

$\mathrm{Na}$ exibição do aviso existem dois botões, quando o botão "Lembrar" é acionado, o aviso será programado para ser reexibido após cinco minutos, já quando o botão "Confirmar" é acionado, o aplicativo verifica se ainda existem visualizações disponíveis para o aviso, caso exista, o aviso será programado para ser exibido após o intervalo informado em seu cadastro. Após a confirmação do aviso, o aplicativo gera um histórico contendo a data e a hora em que a exibição ocorreu, o histórico é gravado no banco de dados local do smatphone e também enviado para o banco de dados do dispositivo do responsável através do Firebase Cloud Messaging. A Figura 11 mostra a representação da tabela responsável pelo armazenamento do histórico do aviso, enquanto a Figura 12 apresenta a estrutura do arquivo JSON para o tráfego do histórico de exibições do aviso, e a Figura 13 apresenta um exemplo da tela de consulta do histórico de exibições do aviso.
Figura 11. Representação da tabela "historicoAviso".

\begin{tabular}{|l|}
\hline \multicolumn{1}{|c|}{ historicoAviso } \\
\hline - _id : Integer \\
- idAviso : Integer \\
- dataExibicao: Text \\
- horaExibicao : Text \\
\hline
\end{tabular}

Figura 12. Estrutura do arquivo JSON para envio do histórico de exibições.

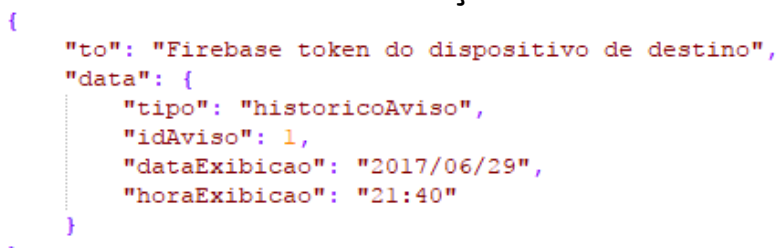

Figura 13. Tela de consulta do histórico de exibições do aviso.

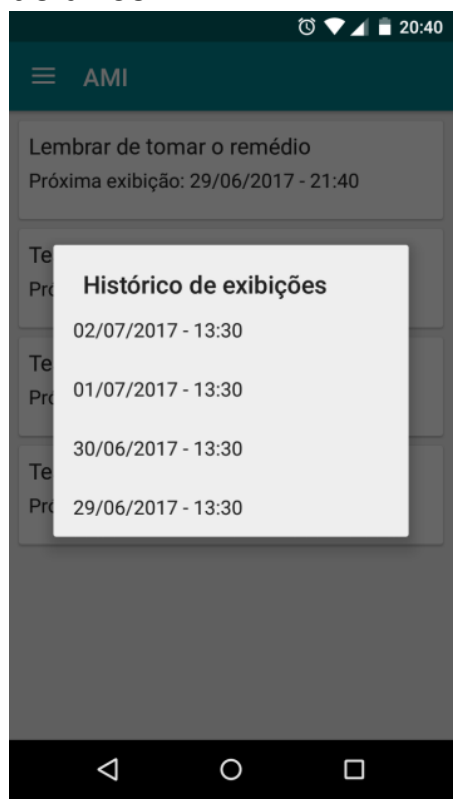

\subsection{Vídeo chamada}

A vídeo chamada é feita utilizando a integração com a API do Skype, sendo necessário que o Skype esteja instalado e configurado no smartphone que realizará a chamada. A API do Skype permite que a chamada seja realizada através de um objeto da classe "SkypeApi", sendo necessário apenas informar o nome de usuário do Skype da pessoa que será chamada, a partir deste ponto a chamada é realizada diretamente pelo aplicativo do Skype. A Figura 14 
apresenta a forma como a chamada é realizada pelo aplicativo.

Figura 14. Método para realização da chamada pela SkypeApi.

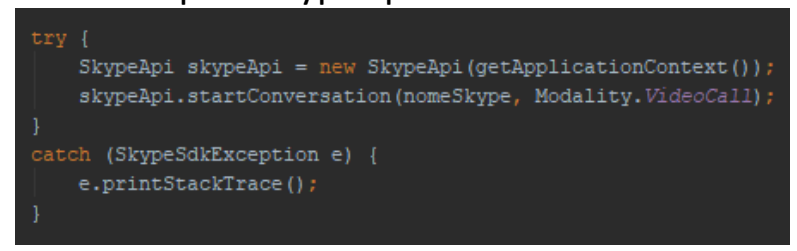

\subsection{Consulta a localização do idoso}

Ao acessar a opção de consultar a localização do idoso, é apresentada uma nova tela no aplicativo. Esta tela contém o Google Maps onde será mostrada a última localização do idoso, através dos valores da latitude e longitude que foram armazenados no Firebase Realtime Database da Google, que permite armazenar dados em nuvem como objetos JSON. Em seguida é enviado um Firebase Cloud Messaging para 0 dispositivo do idoso solicitando a localização atual, assim que o aparelho do idoso receber a solicitação. Imediatamente é solicitado ao GPS do smartphone a sua localização atual, após a posição atual ser reconhecida, o aplicativo atualiza o Firebase Realtime Database com os valores da latitude e longitude do dispositivo. Como o Firebase Realtime Database trabalha com atualizações em tempo real, o dispositivo do responsável mostrará a nova localização imediatamente após os novos valores serem atualizados. A Figura 15 apresenta a estrutura do arquivo JSON de solicitação da localização, e a Figura 16 apresenta a tela de consulta da localização do idoso.

Figura 15. Estrutura do arquivo JSON para envio da solicitação de localização.

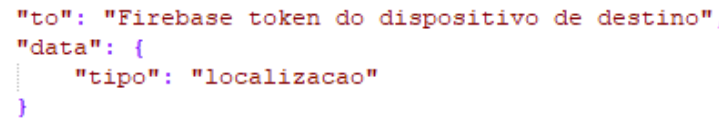

Figura 16. Tela de consulta à localização do idoso.

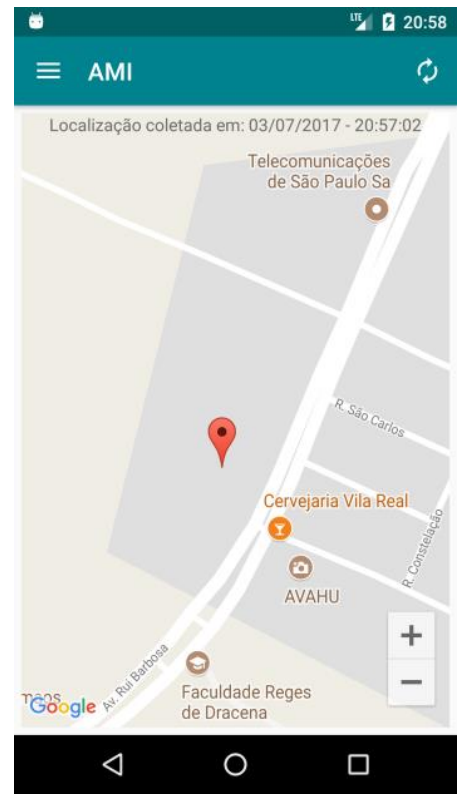

\subsection{Detector de quedas}

$\mathrm{O}$ detector de quedas identifica uma possível queda analisando os valores do sensor acelerômetro ${ }^{4}$ presente no smartphone. $\mathrm{O}$ acelerômetro mede a aceleração do dispositivo em três eixos ( $\mathrm{X}, \mathrm{Y}$ e Z). A Figura 17 mostra a representação dos eixos do acelerômetro em um smartphone.

Figura 17. Representação dos eixos do acelerômetro.

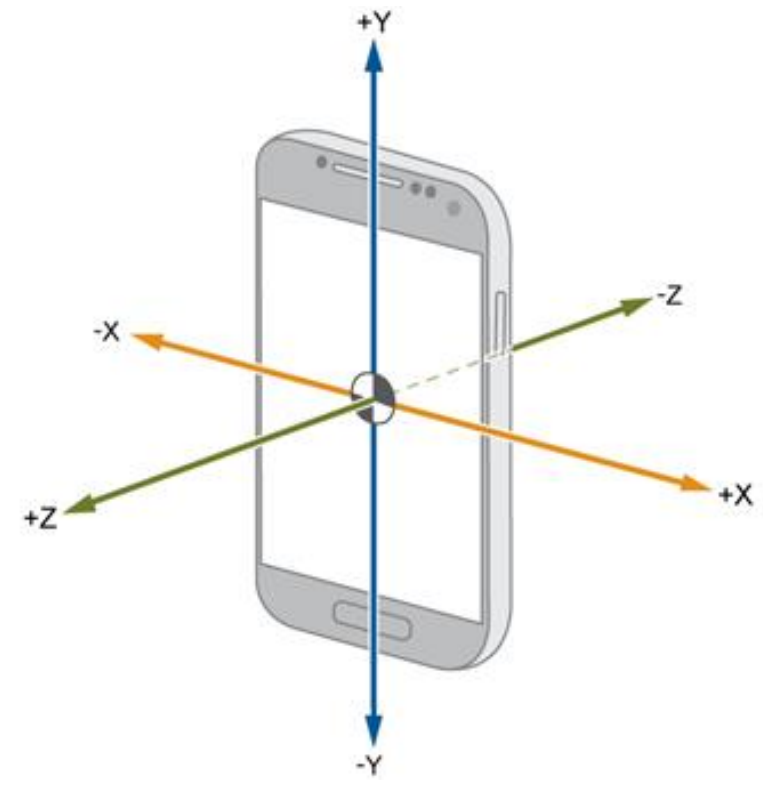

\footnotetext{
${ }^{4}$ Acelerômetro - É um instrumento capaz de medir a aceleração sobre objetos (PRADA, 2009).
} 
Para que as quedas pudessem ser identificadas, primeiramente foi realizada a coleta de dados das variações dos eixos do acelerômetro durante a simulação de atividades diárias normais e de tipos diferente de quedas. Para a coleta dos dados, foi desenvolvido outro aplicativo para smartphones que foi utilizado apenas para coletar os dados, este aplicativo auxiliar armazenava os valores dos três eixos do acelerômetro durante dez segundos após o início da coleta dos dados. Para realizar os testes, o smartphone foi colocado no bolso do autor do trabalho enquanto eram simuladas as atividades diárias normais (caminhar, deitar e levantar, e sentar e levantar), e para analisar o comportamento do acelerômetro, foram simulados quatro tipos de quedas (queda frontal, queda de costas, queda para a lateral direita e queda para a lateral esquerda). Após a coleta dos dados, o aplicativo gerava um arquivo $\mathrm{CSV}^{5}$ que foi enviado para um computador e analisado através da geração de gráficos no Microsoft Excel. Ao todo foram feitas três coletas para cada tipo de atividade normal e de queda. Os Resultados obtidos através das coletas estão descritos na seção 5 .

A rotina de análise do acelerômetro executa em um serviço no sistema operacional do smartphone, pois assim é possível que o monitoramento funcione mesmo que o aplicativo não esteja em execução, quando o valor de um dos eixos atinge um nível característico de uma queda, o aplicativo emite um som de alerta e exibe uma mensagem para o idoso informando que identificou uma possível queda, caso o idoso informe que está bem, a mensagem é simplesmente fechada e o monitoramento retomado. Porém, caso o aplicativo não tenha uma resposta em até quinze segundos, é enviado um SMS para o responsável informando a queda do idoso, e coletada a localização do dispositivo e atualizada no

\footnotetext{
${ }^{5}$ CSV - É um arquivo de planilha que contém dados em cada linha separados por um caractere de separação (em geral uma vírgula ou um ponto e vírgula) (CCM, 2017).
}

Firebase Realtime Database, para que o responsável possa consultar.

\section{EXPERIMENTOS}

Neste trabalho estão apresentados experimentos para verificar a eficácia do recurso criado, se as ferramentas estão funcionando de maneira satisfatória para o seu propósito.

Para os experimentos foi utilizado um smartphone da marca Motorola e modelo Moto $\mathrm{G}$ executando a versão 5.1 do Android e o emulador do SDK do Android simulando um dispositivo da marca $L G$ e modelo Nexus 5 executando a versão 6.0 do Android. O aplicativo batizado de AMI (Aplicativo Monitorador de Idosos), que foi desenvolvido neste trabalho, foi instalado nos dois dispositivos, no emulador ele foi configurado como sendo o smartphone do responsável enquanto o dispositivo físico foi configurado como sendo do idoso.

Para a execução dos experimentos foram realizados testes em todas as funcionalidades do aplicativo, os testes executados estão dispostos na sequência:

- Aviso de exibição instantânea: Foram enviados três avisos instantâneos a partir de cada um dos dois dispositivos, o primeiro aviso foi enviado com a tela do destinatário ligada, o segundo com a tela do destinatário desligada, para testar se a tela seria ligada automaticamente para a exibição do aviso e o terceiro com o destinatário desconectado da internet, para testar se após a reconexão com a internet $\mathrm{o}$ aviso seria recebido;

- Aviso de exibição automática: Foram cadastrados três avisos automáticos, o primeiro foi cadastrado para ser exibido uma única vez às 12 horas do dia 01/07/2017, o segundo foi cadastrado para ser exibido oito vezes com um intervalo de doze horas entre cada exibição, a primeira exibição foi configurada para ocorrer às 9 horas do dia 29/06/2017, já o terceiro aviso 
foi configurado para ser exibido quatro vezes com um intervalo de um dia entre cada exibição, a sua primeira exibição foi configurada para ocorrer às 13 horas e 30 minutos do dia 29/06/2017;

- Vídeo chamada: Para este teste não foi possível utilizar o emulador, pois o mesmo não possui o aplicativo da Google Play Store e assim não seria possível a instalação e execução do Skype, a chamada foi feita a partir do dispositivo do idoso e a conta do Skype recebedora da chamada foi configurada em um segundo dispositivo físico, utilizado apenas para este teste;

- Consulta a localização do idoso: Este teste consistiu apenas em acessar a tela para consulta de localização no dispositivo do responsável e aguardar até que o smartphone do idoso recebesse o pedido de localização e atualizasse 0 Firebase Realtime Database após coletar os dados do GPS, para que a localização atual fosse apresentada ao responsável;

- Detector de quedas: Este teste consistiu em realizar simulações de atividades diárias normais e quedas pelo autor do trabalho. As simulações consistiram em realizar uma caminhada de 100 metros e repetir três vezes cada uma das outras atividades normais (deitar e levantar, e sentar e levantar) e as quedas (frontal, costas, lateral esquerda e lateral direita); Após o alerta de cada queda ser exibido foi deixado que o aplicativo enviasse o SMS para o responsável. Para o envio do SMS, foi configurado o número do smartphone que estava configurado como sendo do idoso, pois não seria possível enviar para o emulador.

\section{RESULTADOS}

As coletas das variações do acelerômetro através do aplicativo auxiliar serviram como base para a rotina de análise e detecção de quedas. A Figura 18 mostra a representação dos dados coletados durante a simulação de uma caminhada, enquanto a Figura 19 mostra a representação dos dados coletados durante a simulação de uma queda frontal.

Figura 18. Variações do acelerômetro durante uma caminhada.

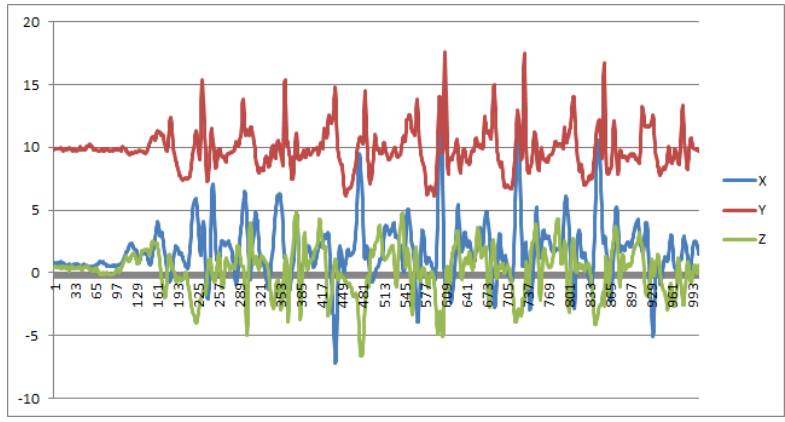

Figura 19. Variações do acelerômetro durante uma queda frontal.

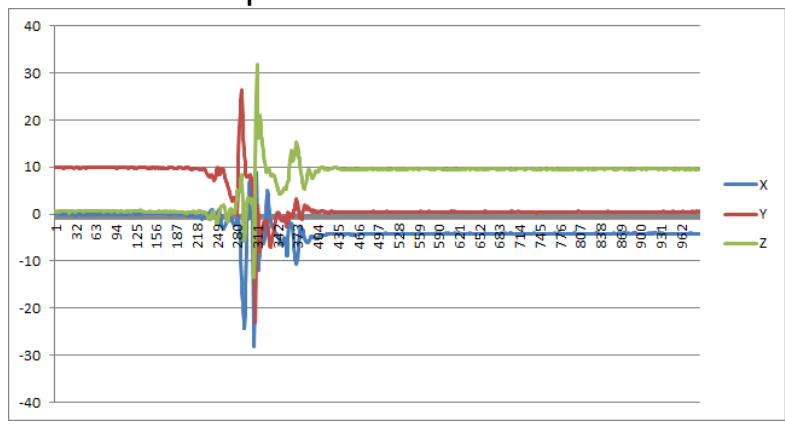

$\mathrm{Na}$ análise dos dados, foi identificado que para atividades normais os três eixos se mantinham dentro de uma faixa de valores, tanto em valores positivos quanto em negativos, e que para quedas os valores positivos alcançavam números mais altos e os negativos números mais baixos, formando assim uma faixa de valores mais larga. Após esta percepção, foram isolados o menor e o maior valor de cada eixo dentro de todas as atividades normais, assim como em todas as quedas, obtendo assim os extremos de cada eixo para os dois tipos de eventos simulados. Após os extremos serem isolados, foram calculadas as médias entre a diferença de cada extremo e decidido que qualquer valor que ultrapassasse a média calculada seria reconhecido pelo aplicativo como uma possível queda. A Figura 20 apresenta os 
extremos de cada eixo, assim como a média calculada que foi utilizada para identificar os valores que representavam uma possível queda.

Figura 20. Representação gráfica da análise feita nos dados.

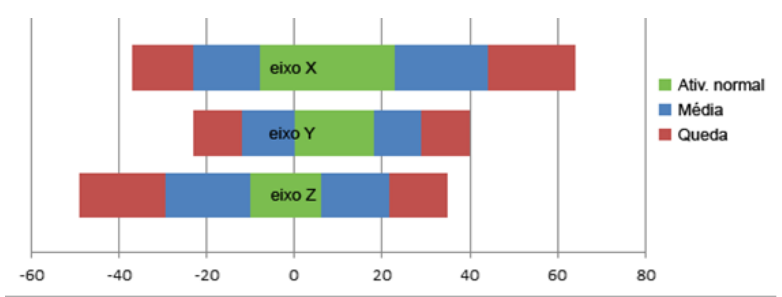

Com os resultados gerados a partir dos dados coletados com o aplicativo auxiliar, foi possível desenvolver o detector de quedas, porém, nos experimentos realizados ficou constatado que o detector de quedas possui algumas limitações, o aplicativo conseguiu detectar apenas uma das três simulações de queda lateral para a direita, o motivo era que o smartphone sempre foi colocado no bolso esquerdo do autor do trabalho que realizou as simulações, com isso no momento da queda o dispositivo sofre um impacto menor e o acelerômetro varia menos do que em outros casos, já nos outros tipos de queda o aplicativo conseguiu detectar todas as simulações, sempre enviando o SMS para o responsável. Outra limitação no detector de quedas ocorreu por uma característica do smartphone utilizado nos testes, alguns modelos desativam 0 acelerômetro após a tela ser desligada, sendo necessário que a tela esteja ativa para que o detector possa funcionar, o que nem sempre ocorrerá no ambiente acobertado pelo recurso.

Os demais experimentos com os outros recursos do aplicativo ocorreram de maneira muito satisfatória, exibindo todos os avisos de acordo com o programado, o dispositivo do idoso consultou a sua localização e disponibilizou para o responsável consultar assim que a requisição foi recebida e a vídeo chamada com o Skype foi realizada sem problemas através da solicitação do aplicativo.

\section{CONCLUSÃO}

Com este trabalho foi possível perceber que, mesmo com algumas limitações, os recursos tecnológicos presentes nos smartphones podem ser utilizados como ferramentas para auxiliar e monitorar pessoas idosas que moram sozinhas, e assim contribuir para sua segurança e saúde, além de passar uma tranquilidade para seus responsáveis.

É importante ressaltar que o recurso desenvolvido neste trabalho deve ser utilizado apenas como uma ferramenta auxiliar, e não dispensa a ajuda presencial dos responsáveis ou de profissionais cuidadores de idosos.

Para trabalhos futuros, poderiam ser feitas melhorias no detector de quedas, utilizando um acelerômetro externo que ficaria preso ao corpo do idoso através de um bracelete ou outro material que o mantenha fixo, evitando assim o problema ocasionado com o não funcionamento do acelerômetro quando a tela do smartphone é desativada. Poderiam ser feitos também testes com um grupo fechado de pessoas idosas, com limitações físicas e motoras diferentes entre eles, proporcionando assim uma melhor avaliação do comportamento do recurso.

\section{REFERÊNCIAS}

BEZERRA, F. C; ALMEICA, M. I; NÓBREGATHERRIEN, S. M. Estudo sobre envelhecimento no Brasil: revisão bibliográfica. Revista Brasileira de Geriatria e Gerontologia, Rio de Janeiro, 2012.

CCM. Arquivo CSV. Disponível em: <http://br.ccm.net/faq/5972-arquivo-csv>. Acesso em: 03 jul. 2017.

\section{COLLUCl, C. Número de idosos que moram sozinhos triplica em 20 anos. 2013. Disponível em: <http://www1.folha.uol.com.br/equilibrioesa ude/2013/12/1389765-numero-de-idosos-}


que-moram-sozinhos-triplica-em-20anos.shtml>. Acesso em: 02 fev. 2017.

JSON.org. Introdução ao JSON. Disponível em: <http://www.json.org/json-pt.html>. Acesso em: 03 jul. 2017.

LIMA, M. R. et al. Utilização dos recursos do IOS para monitorar pessoas de terceira idade na prática de atividades físicas. Colloquium Exactarum, Presidente Prudente, v. 5, n. 2, p. 12-29, 2013.

MAIA, B. C. et al. Consequências das quedas em idosos vivendo na comunidade. Revista Brasileira de Geriatria e Gerontologia, Rio de Janeiro, v. 14, n. 2, p. 381-393, 2011.

MIRANDA, A.; MARIK, P. Sistema Detector de Quedas (SDQ). Curitiba: Pontifícia Universidade Católica do Paraná, 2008.

PRADA, R. O que é um acelerômetro. 2009. Disponível em: <https://www.tecmundo.com.br/curiosidade /2652-o-que-e-um-acelerometro-.htm>. Acesso em: 03 jul. 2017.

SANTOS, J. A importância de tomar remédio na hora certa. 2011. Disponível em: <http://citrus.uspnet.usp.br/aun/exibir?id=4 $212 \&$ ed $=705 \& f=18>$. Acesso em: 24 mar. 2015.

SILVEIRA, F. Broadcast Receiver - sua aplicação respondendo a eventos. 2015. Disponível em: <http://www.felipesilveira.com.br/2015/04/ broadcast-receiver-fazendo-sua-appresponder-a-eventos/>. Acesso em: 12 ago. 2017.

SQLite.org. Sobre o SQLite. Disponível em: <https://www.sqlite.org/about.html>. Acesso em: 03 jul. 2017.

TANJI, F. T. K. et al. Uso do Kinect para monitoramento de exercícios físicos realizados por pessoas de terceira idade.
Colloquium Exactarum, Presidente Prudente, v. 6, n. 1, p. 99-113, 2014. 